\title{
Risk factors and outcomes of carbapenem-resistant Klebsiella pneumoniae infections
}

\author{
Eleonora Pistella, Claudio Santini \\ Complex Operative Unit of Internal Medicine, Vannini Hospital, Roma, Italy
}

\begin{abstract}
In the nosocomial setting, antimicrobial-resistant Enterobacteriaceae are a growing challenge, and alarming trends in resistance are currently reported all over the world. Isolates of Enterobacteriaceae producing ampC $\beta$-lactamases and extended spectrum $\beta$ lactamases are endemic in many hospitals, and are frequently resistant also to other classes of antibiotics, such as fluoroquinolones and aminoglycosides. The risk of infections due to multi-drug resistant strains should be considered also for outpatients who have had recent contact with the health system. Both nosocomial and health-care associated infections should be treated with a combination of antibiotics active against multi-drug resistant Gram negative and methicillin-resistant Staphylococcus aureus. In the absence of effective antimicrobial stewardship programs, this aggressive therapeutic approach might lead to abuse of broad-spectrum antibiotics, with consequent increase in resistances. To contain the possible antibiotic overuse, several decisional strategies, often based on risk-score systems supporting the clinical decisions, have been proposed. In this context of high antibiotic selection pressure, carbapenem-resistant pathogens recently began to spread in many hospitals. Carbapenem-resistant Klebsiella pneumoniae, as well as carbapenem-resistant Acinetobacter baumannii and Pseudomonas aeruginosa, represent the new major challenges to patient safety. Against these organisms the initial empiric treatment is generally ineffective. The poor clinical outcome associated with carbapenem-resistant $K$. pneumoniae infections is probably due to the delete in the beginning of an appropriate antibiotic treatment, rather than to the increased virulence of pathogens. Only few therapeutic options are available, including colistin, tigecycline, aminoglycosides and carbapenems in selected cases. Several combinations of these antibiotics have been used, but no ideal regimen has been currently established.
\end{abstract}

\section{Aims of the work}

Based on the evidence reported in the literature the aims of the work are: i) to evaluate the risk factors and the new score-system approaches to correctly identify the patients at risk for multi-drug resistant infections and, in particular, for carbapenem-resistant Klebsiella pneumoniae infections; ii) to correlate the different clinical conditions and therapeutic options to the outcome of carbapenem-resistant K. pneumoniae infections.

Correspondence: Claudio Santini, Complex Operative Unit of Internal Medicine, Vannini Hospital, via Acqua Bullicante 4, 00177 Roma, Italy.

E-mail: santini.cla@gmail.com

Key words: Carbapenem-resistant Klebsiella pneumoniae; multi-drug resistant Enterobacteriaceae; nosocomial infections.

Received for publication: 6 October 2016.

Accepted for publication: 20 October 2016.

This work is licensed under a Creative Commons Attribution NonCommercial 4.0 License (CC BY-NC 4.0).

(C) Copyright E. Pistella and C. Santini, 2016

Licensee PAGEPress, Italy

Italian Journal of Medicine 2016; 10:339-348

doi:10.4081/itjm.2016.798

\section{Epidemiology of carbapenem-resistant Klebsiella pneumoniae}

Hospital-acquired infections (HAIs) occur in 4.5\% of hospital admissions and cause almost 99,000 deaths, representing the sixth cause of death in USA. The annual HAIs-related costs are estimated of 30 billion US dollars. One third of HAIs are preventable. ${ }^{1}$ HAIs are often related to medical devices (i.e., central venous catheter, urinary catheter or mechanical ventilator) or complicate surgical procedures. Urinary tract infections are by far the most common HAIs, but bloodstream infections and lower respiratory tract infections are the most lethal.

Gram-negative bacteria are responsible for more than one third of HAIs, and predominate in case of ventilator-associated pneumonia (47\%) and urinary tract infections (45\%). ${ }^{1}$ In most Intensive Care Units (ICUs), Gram negative bacteria are the leading pathogens. In USA, $70 \%$ of HAIs occurring in ICUs are due to Gramnegative bacteria. ${ }^{2}$ As shown in Table 1 , in Italian ICUs, Gram negative bacteria cause more than half of bloodstream infections, pneumonia and urinary tract infections. Klebsiella spp. in particular, has been reported as the leading pathogen for both bloodstream infections and ventilator-associated pneumonia. ${ }^{3}$

For many decades, several classes of antibiotics (third generation cephalosporins, ureidopenicillins 
plus $\beta$-lactamase inhibitor, fluoroquinolones and aminoglycosides) ensured a highly effective treatment for severe Gram-negative infections. Unfortunately, Gram-negative bacteria are highly efficient in acquiring and spreading genes that code for mechanisms of antibiotic resistance, especially when exposed to antibiotic pressure. Since mid-1980s, a growing number of Gram-negative bacilli, mainly Enterobacteriaceae, acquired the ability to produce a new group of enzymes, the extended-spectrum $\beta$-lactamases (ESBLs). ESBLs are capable of hydrolyzing penicillins, third generation cephalosporins and monobactams. ESBLs are often located on plasmids and easily spread from strain to strain and between bacteria species. ESBLproducing strains are now endemic in many hospitals all over the world, representing a universal publichealth concern. ${ }^{4}$ Furthermore, the plasmids carrying ESBL genes often carry other genes for antibiotic resistance such as AmpC $\beta$-lactamases or genes that code for aminoglycosides and fluoroquinolones resistance. The organisms that show in vitro resistance to three or more classes of antibiotics are defined as multi-drug resistant (MDR). In Italy, from 2011 to 2014 , the incidence of resistance to third generation cephalosporins increased from $19.8 \%$ to $28.7 \%$ among Escherichia coli isolates and from $15.9 \%$ to $56.5 \%$ among Klebsiella pneumoniae isolates. ${ }^{5}$ In the same period, the rate of MDR strains (combined resistance to fluoroquinolones, third generation cephalosporins and aminoglycosides) among $E$. coli and $K$. pneumoniae isolates increased from 10.3 to $13.8 \%$ and from 32.9 to $44 \%$ respectively. ${ }^{5}$ Against infections caused by MDR Gram negative bacteria the therapeutic options are restricted to few agents and poor clinical outcomes are more frequent. ${ }^{6-8}$ Carbapenems are regarded as the drugs of choice for treatment of suspected or proven serious infections caused by ESBL-producing and MDR organisms. Nevertheless, the increased use of carbapenems contributed to the emergence of carbapenem-resistant strains, which actually represent the main concern in the treatment of Gram-negative infections. K. pneumoniae, among Enterobacteriaceae family, is the species more frequently involved in this phenomenon.

The first strain of carbapenem-resistant $K$. pneumoniae (CRKP) was isolated in 1996 in North Carolina (USA). It was a strain of $K$. pneumoniae producing a $\beta$-lactamase able to hydrolyze carbapenems and even $\beta$-lactamase inhibitors, as well as penicillins, broadspectrum cephalosporins (including cefepime) and monobactam. ${ }^{9}$ The enzyme was named $K$. pneumoniae carbapenemase (KPC). In the subsequent few years many carbapenem-resistant strains of $K$. pneumoniae and, more marginally, of other species of Enterobacteriaceae were detected in several hospitals in New York City ${ }^{10}$ and rapidly spread in many US states.

With the exception of sporadic detections, the phenomenon of CRKP infections in Europe began more than ten years later. From 2009 to 2013 a significant increasing trend in CRKP nosocomial infections was observed. In 2014 the mean percentage of carbapenemresistant strains among K. pneumoniae isolates in Europe was $7.3 \%$. However, in three countries (Greece, Italy and Romania) the reported percentage of car-

Table 1. Percentage of the most frequently isolated bacteria in Intensive Care Unit-acquired infections in Italy, 2012.

\begin{tabular}{|c|c|c|c|}
\hline & Bloodstream infections & Ventilator associated pneumonia & Urinary tract infections \\
\hline Staphylococcus aureus & 8.7 & 14.6 & - \\
\hline Coag. neg. staphylococci & 13.6 & - & - \\
\hline Enterococcus spp. & 9.8 & 2.6 & 21.7 \\
\hline Gram-positive & 32.1 & 17.2 & 21.7 \\
\hline Klebsiella spp. & 15.6 & 16.9 & 4.3 \\
\hline Escherichia coli & 7.4 & 7.5 & 34.8 \\
\hline Enterobacter spp. & 5.5 & 5.9 & - \\
\hline Serratia spp. & 3.8 & 3.0 & - \\
\hline Morganella spp. & - & - & 8.7 \\
\hline Proteus spp. & - & - & 4.3 \\
\hline Pseudomonas aeruginosa & 11.2 & 16.8 & 4.3 \\
\hline Acinetobacter spp. & 9.2 & 13.2 & 4.3 \\
\hline Stenotrophomonas maltophilia & - & 2.4 & - \\
\hline Gram-negative & 52.7 & 65.7 & 60.7 \\
\hline
\end{tabular}

Data from European Center for Disease Prevention and Control (ECDC), 2015. ${ }^{3}$ 
bapenem-resistance among $K$. pneumoniae isolates was considerably higher than in any other country $(62.3 \%$, $32.9 \%$ and $31.5 \%$ respectively). These countries also reported the highest level of MDR strains (combined resistance to fluoroquinolones, third generation cephalosporins and aminoglycosides). ${ }^{5}$ The trend of antibiotic resistance of $K$. pneumoniae isolates in Italy in the period 2011-2014 is reported in Table 2. The CRKP isolates are usually MDR and are susceptible only to colistin, tigecycline and one or more aminoglycoside.

\section{Key messages}

- Gram-negative bacteria cause one third of HAI

- In Italy K. pneumonia nosocomial isolates are: i) resistant to $3^{\text {rd }}$ generation cephalosporins: $56.5 \%$; ii) MDR: 44\%; and iii) resistant to carbapenems: $32.9 \%$.

\section{Risk factors for multi-drug resistant infections}

A large part of the hospitalized patients, and in particular of the patients admitted to the Internal Medicine wards, are old, with several comorbidities, recurrent hospitalizations and very frail. Many infections occurring in these patients are severe and require an early empiric treatment, considering that the most important prognostic factor for severe infections is the early onset of an effective treatment. ${ }^{11}$ Therefore, in the selection of appropriate antibiotic regimen, it is essential to recognize the risk of the patient for MDR infections. The site of acquisition of infection (community, hospital or nursing-home), the pharmacologic anamnesis for previous antibiotic exposure, the comorbidities and the condition of immunosuppression, as well as the site and severity of infection, determine the choice of empiric antibiotic regimens.

\section{Nosocomial acquisition}

Community acquired infections (CAIs) are rarely due to MDR strains. Nosocomial acquisition repre- sents the most important risk factor for MDR infections, and in particular for CRKP infections. Several reports compared the incidence of resistant isolates among pathogens responsible of CAIs and HAIs. In the series of 61 intra-abdominal infections reported by Boontham et al., ESBL-producing stains were present in $15.6 \%$ of CAIs and in $68.8 \%$ of HAIs. ${ }^{12} \mathrm{Mc}$ Kay recently reported a series of 740 bloodstream infections, nearly three-quarter of which were hospitalacquired. Enterobacteriaceae caused $39.2 \%$ of both HAIs and CAIs; the community-acquired Enterobacteriaceae were highly susceptible to antibiotics (ceftriaxone $96.1 \%$, ciprofloxacin $92.2 \%$, gentamicin 95.6\%), while the hospital-acquired Enterobacteriaceae were significantly less susceptible (58.5\%, $70 \%$, and $64.6 \%$ respectively). ${ }^{13}$ During hospitalization, the upper respiratory tract and the gastrointestinal tract become colonized by nosocomial microorganisms. Colonization frequently progresses to microaspiration, tissue invasion and infection. Gastro-intestinal tract, in particular, became the main reservoir of antibiotic-resistant pathogens, such as CRKP, Clostridium difficile and Candida spp. that currently spread in the wards of many hospitals causing a large number of nosocomial outbreaks. All infections developing more than $48 \mathrm{~h}$ after hospital admission are defined as HAIs and must be considered as possibly due to MDR strains. The risk of MDR infections is also related to the length of hospitalization. In 2012 in USA the rate of infections due CRKP resulted $4.6 \%$ in the short-term acute-care hospitals and $17.8 \%$ in the long-term acute-care hospitals. ${ }^{14}$ Hospitalized patients are usually exposed to one or more other risk factors for MDR infections, such as antibiotic treatments, use of prosthetic devices, surgical procedures or compromised functional status.

The phenomenon of antibiotic resistance in the hospitals is primarily due to the widespread use of antibiotics, often for prolonged periods of time. Antimicrobial stewardship programs play a critical role to improve appropriate antibiotic use and to reduce the emergence of further resistance. ${ }^{11}$

Table 2. Percentage of resistant isolates of Klebsiella pneumoniae in Italy in the period 2011-2014.

\begin{tabular}{lllll}
\hline Class of antibiotics & $\mathbf{2 0 1 1}$ & $\mathbf{2 0 1 2}$ & $\mathbf{2 0 1 3}$ & $\mathbf{2 0 1 4}$ \\
\hline Fluoroquinolones & 45.7 & 49.9 & 54.4 & 55.7 \\
\hline III-generation cephalosporins & 45.9 & 47.9 & 55.1 & 56.5 \\
\hline Aminoglycosides & 34.6 & 42.4 & 44.9 & 48.6 \\
\hline MDR* $^{*}$ & 32.9 & 40.2 & 41.8 & 44.0 \\
\hline Carbapenems & 26.7 & 29.1 & 34.3 & 32.9 \\
\hline
\end{tabular}

MDR, multi-drug resistant. *Combined resistance to fluoroquinolones, III-generation cephalosporins and aminoglycosides. Data from European Center for Disease Prevention and Control (ECDC), 2015. 


\section{Healthcare-associated infections}

The risk of infections due to MDR strains involves not only hospitalized patients, but also outpatients who have had recent contact with the health system. The term of healthcare-associated infections (HCAIs) was originally used to classify pneumonia occurring in patients with at least one of the following risk factors: residence in a nursing home, recent hospitalization (more than 2 days in the previous 3 months), prior antibiotic exposure, immunosuppression, chronic hemodialysis. ${ }^{15}$ The concept of HCAIs was created to help clinicians to identify these patients and to treat their infections as HAIs, with empiric regimens consisting in the combination of antibiotics active against Pseudomonas aeruginosa and methicillin-resistant Staphylococcus aureus. ${ }^{16}$ However, a great number of patients admitted to the hospital possess one or more of the above-mentioned characteristics and the selection of antibiotic regimens based on the concept of HCAIs might lead to the overuse of broad-spectrum antibiotics, with consequent increase in resistance. Venditti et al. reported that among patients with pneumonia presenting to the Emergency Department, $50 \%$ met criteria for HCAI, but the incidence of resistant organisms ranged from 10 to $30 \% .{ }^{17}$ Shorr et al. ${ }^{18}$ and Nseir et al. ${ }^{19}$ investigated several risk factors for developing MDR infection to identify their specific predictive values. Multivariate analysis identified recent hospitalization [odds ratio (OR) 4.2 and 3.9 respectively], nursing-home residence (OR 2.7 and 2 respectively), prior antibiotic therapy (OR not evaluated and 2.3 respectively) and hemodialysis (OR 2.11 and not evaluated respectively) as independent risk factors for MDR infection.

To reduce the risk of abuse of antibiotics, new approaches, based on the utilization of decisional riskscores have been proposed for a more precise selection of the treatment of healthcare-associated pneumonia and HCAIs. In the clinical score proposed by Shorr et al., the points are assigned as follows: 4 for recent hospitalization, 3 for residence in nursing-home, 2 for chronic hemodialysis, 1 for ICU admission within 24 hours of evaluation in Emergency Department, for a maximum score of 10 . The prevalence of resistant pathogens is approximately $15 \%$ with a score of 0 , and increases to $60 \%$ with a score $>6 .{ }^{18}$ In 2013 , Shindo el al. individuated six independent risk factors for MDR in patients with community acquired pneumonia and healthcare-associated pneumonia: prior hospitalization, previous antibiotic use, immunosuppression, use of gastric acid-suppressive agents, tube feeding and non-ambulatory status. The risk for MDR infection is high in the presence of three or more risk factors. ${ }^{20}$ In 2013, Maruyama el al. proposed a therapeutic algorithm based on the presence of one major criterion, represented by the severity of illness (defined as need for mechanical ventilation or ICU admission) and four minor criteria represented by risk factors for MDR pathogens (hospitalization for more than 2 days in the past 90 days, antibiotics in the past 180 days, poor functional status with Barthel Index $<50$ and immunosuppression). They treated with regimens for hospital-acquired pneumonia only the patients with two or more minor criteria. Almost half of the patients with healthcare-associated pneumonia (according to classic definition) had less than two criteria and were treated as community-acquired pneumonia; the treatment resulted inappropriate in only $7.1 \%$ of patients. ${ }^{21}$ In 2015 , Falcone et al. evaluated the risk factors for MDR among 900 patients admitted for pneumonia and proposed the ARUC (Assessment of Risk of multidr $U \mathrm{~g}$ resistant pathogens in Community-onset pneumonia) score, in which the points are assigned as follows: 1 point in the presence of at least one criterion for healthcare-associated pneumonia (previous hospitalization in the last 3 months, dialysis, intravenous therapy in the previous 30 days, residence in nursing-home), 0.5 points for bilateral pulmonary infiltration, 0.5 points for pleural effusion and 1.5 point if $\mathrm{PaO}_{2} / \mathrm{FiO}_{2}<300$. When the score was $<0.5$, the prevalence of MDR pathogens was very low (3\%), while the prevalence climbed to $41 \%$ when the score was $\geq 3$. As screening test, the ARUC score has been validated on an external cohort of patients hospitalized for pneumonia demonstrating a good sensitivity (87\%) and specificity $(70 \%))^{22}$ However, although the promising results of a better selection of the patients at risk for MDR and of a possible reduction in the use of antibiotics, currently these risk-score approaches have been validated only for specific disease (i.e., healthcareassociated pneumonia).

\section{Residence in nursing home}

Nursing home are recognized as a reservoir of MDR bacteria. Colonization by MDR Gram negative bacteria of enteric origin has been detected in $51 \%$ of residents in long-term care facilities, especially if affected by advanced dementia or confined in the bed. ${ }^{23}$ In patients residing in nursing home is very high the risk of aspiration pneumonia, because of frequent deterioration of consciousness and swallowing dysfunction. The term of nursing-home-acquired pneumonia (NHAP) has been proposed for this entity. When compared to community-acquired pneumonia, NHAPs have a higher incidence of Gram-negative pathogens $(18.8 \%$ vs $5.5 \%)$ and a four-fold increased mortality. ${ }^{24}$

\section{Prior antibiotic therapy}

Few studies investigated the relationship between previous antibiotic therapy and prevalence of antibi- 
otic resistance. A recent meta-analysis documented that individuals treated with antibiotics in primary care for a respiratory or urinary tract infection frequently develop bacterial resistance to that antibiotic. The effect is the greatest in the month immediately after treatment but may persist for up to 12 months (OR 2.4). This effect not only increases the population carriage of organisms resistant to first line antibiotics, but also creates the conditions for increased use of second line antibiotics in the community. ${ }^{25}$

\section{Hemodialysis}

Patients undergoing chronic hemodialysis are at high risk for colonization with MDR strains. These patients access three time per week the dialysis unit, a close area where cross-transmission of MDR organisms is highly probable. Moreover, many of them get other risk factors, as recent hospitalization, recent antibiotic therapy or residence in nursing home. Although MDR Gram positive pathogens are prevalent, colonization and infection with MDR Gram-negative bacteria are also frequent. In a prospective study MDR Gram-negative bacilli were recovered from $16 \%$ of outpatients under chronic hemodialysis. ${ }^{26}$ The risk was higher among patients residing in nursing home or with antibiotic exposure in the previous three months. ${ }^{26}$

\section{Key messages}

- The main risk factor for MDR is the hospital acquisition

- In the setting of HCAIs the main individual risk factors are: i) recent hospitalization (OR 4.2); ii) nursing-home residence (OR 2.7); iii) previous antibiotic therapy (OR 2.3); iv) chronic hemodialysis (OR 2.1)

- Risk score approaches are promising, but still not validated for extensive use.

\section{Risk factors for carbapenem-resistant Klebsiella pneumoniae infections}

Many factors associated with carbapenem resistance are related to the species and the enzymatic profile of the isolates. ${ }^{27}$ Other risk factors for colonization or infection by CRKP, and specifically for KPC-producing K. pneumoniae, depend on the patient status. In a large, retrospective, matched case-control study performed in 2014 in five Italian hospitals, Tumbarello et al. investigated the following conditions: comorbidities (Charlson score, hematologic cancer, neutropenia), previous contacts with the health system (previous hospitalization, previous admission to ICU), presence of devices (urinary catheter, central venous catheter, surgical drainage) and recent use of antibiotics (in particular carbapenems and fluoroquinolones). Eight independent risk factors for isolation of KPC-producing K. pneumoniae (colonization and infection) were identified: $\geq 2$ previous (within 12 months) acute-care hospitalizations (OR 5.92, $\mathrm{P}<0.001$ ), previous (within 12 months) admission to ICU (OR 5.13, P<0.001), indwelling urinary catheter (OR 3.89, $\mathrm{P}<0.001$ ), recent (within 30 days) carbapenem therapy (OR 2.98, $\mathrm{P}<0.001$ ), recent (within 30 days) fluoroquinolones therapy (OR 1.69, $\mathrm{P}<0.001$ ), indwelling central venous catheter (OR 1.66, $\mathrm{P}<0.001$ ), hematologic cancer (OR 1.90, $\mathrm{P}=0.002)$ and surgical drain (OR 1.62, $\mathrm{P}=0.004)$. Considering the subgroup of true infections, the recognized risk factors were: Charlson score $\geq 3$ (OR 7.49, $\mathrm{P}<0.001$ ), $\geq 2$ previous (within 12 months) acute-care hospitalizations (OR 4.26, $\mathrm{P}<0.001$ ), recent (within 30 days) carbapenem therapy (OR 3.59, $\mathrm{P}<0.001$ ), indwelling central venous catheter (OR 2.59, $\mathrm{P}<0.001$ ), recent (within 30 days) fluoroquinolones therapy (OR 2.22, $\mathrm{P}<0.001$ ), recent (within 30 days) surgical procedures (OR 2.03, $\mathrm{P}<0.001)$ and neutropenia (OR 3.19, $\mathrm{P}=0.003$ ). Based on the reported data, the authors proposed a model for evaluating the risk of CRKP isolation and infection. The presence of three or more of the above reported risk factors was associated with a OR of 11.33 for CRKP isolation (specificity: $88 \%$, accuracy: $79 \%$ ) and to a OR of 10.25 for infection (specificity: 90\%, accuracy: 78\%). ${ }^{28}$

Other reports confirm that previous hospitalizations and previous ICU admission are the stronger predictors of CRKP acquisition. ${ }^{29}$ The rate of enteric CRKP colonization in ICUs is actually very high $(>70 \%)$, in particular among patients with prolonged hospitalization. ${ }^{30,31}$ Duration of enteric colonization after discharge has been evaluated in patients recently ( $<4$ months) or remotely ( $\geq 4$ months) colonized. Persistent colonization (CRKP positivity after 3-6 months of follow up) was more common in recently colonized patients $(61 \%)$ than in remote colonized patients (28\%). Risk factors for persistent colonization were the presence of any catheter, the residence in nursing home, and the low functional status. ${ }^{32}$ Persistently colonized patients represent an important reservoir for diffusion of CRKP in healthcare facilities. ${ }^{33}$ Moreover, CRKP infections develop in approximately $9 \%$ of persistently colonized patients. ${ }^{34}$ Colonization by both CRKP and C. difficile has been reported as relatively common in nursing home $(5.7 \%)$ and CRKP sepsis is more frequent in these patients and in patients with recent $C$. difficile infection. ${ }^{35}$ Selective digestive decontamination using oral colistin and/or gentamycin has been evaluated to eradicate the status of CRKP carrier in specific categories of patients (i.e., oncohematologic patients or candidates to solid organ transplantation), but the results were not convincing. The risk of infection was similar between colonized and non-colonized patients, the efficacy was limited to a short time and, more important, a rapid increase in bacterial resistance was observed. 
Many concerns have been raised, in particular, about the selection of colistin-resistant strains. ${ }^{36-38}$

\section{Key message}

Risk factors for CRKP are: i) $\geq 2$ hospitalizations in the previous 12 months; ii) previous ICU admission; iii) indwelling urinary catheter; iv) carbapenem therapy in the previous 30 days; v) fluoroquinolones therapy in the previous 30 days; vi) indwelling central venous catheter; vii) hematologic cancer; viii) surgical drain; ix) enteric CRKP colonization; x) recent $C$. difficile infection.

\section{Selection of initial empiric antibiotic treatment}

Third generation cephalosporins, monobactam and ureidopenicillins plus $\beta$-lactamase inhibitor, in monotherapy or combined to aminoglycosides or fluoroquinolones represented a valid empiric treatment against Gram negative bacteria before the emergence of ESBL-producing Enterobacteriaceae, but are now largely ineffective. The high rate of fluoroquinoloneresistant strains makes unsafe the use of this class of antibiotic in monotherapy for empiric regimens. As a consequence, currently carbapenems are the drugs of choice for the treatment of serious infections due to ESBL-producing strains and also for empiric treatment of severe infections in patients with risk factors for MRD pathogens. Extensive use of carbapenems, however contributes to the emergence of carbapenem-resistant Gram-negative infections. Ureidopenicillins plus $\beta$-lactamase inhibitor can represent an alternative to carbapenems for specific treatment of infections due to microorganisms susceptible when MIC $\leq 2 \mu \mathrm{g} / \mathrm{mL}$ as well as for empiric treatment of urinary infections and nonsevere infections from other sites. ${ }^{39-40}$

Before starting with empiric broad-spectrum antibiotic treatment, all in-patients suspected to have any infection, as well as outpatients admitted for severe infection, should perform a complete microbiological assessment (cultures of blood, urine and endotracheal aspiration if appropriate). The empiric treatment should be re-evaluated after $48 \mathrm{~h}$ and in the event that modified according to clinical response and microbiological results with de-escalation in the absence of resistant strains. De-escalation of the empiric, broad spectrum treatment to specific culture-driven therapy is the most cost-effective strategy to reduce the exposure to antibiotics like carbapenems or vancomycin, that represent the last option against MDR bacteria. ${ }^{40}$ Excluding critically ill patients, de-escalation is suggested in any case in which MDR strains are not recovered. However, the microbiological cultures are frequently not performed and a critical re-evaluation of the empiric treatment is often lacking; as a conse- quence, the strategy of de-escalation therapy is still not widely adopted.

Unfortunately, the therapeutic options for carbapenem-resistant Gram-negative bacteria are very limited and include only colistin, tigecycline and aminoglycosides; meropenem is usefully employed for in vitro resistant strains with MIC values $\leq 8$ (about $30 \%$ of isolates).

Due to the nephrotoxicity of colistin and to the lack of anti-Pseudomonas activity of tigecycline, these two agents are not suitable for empiric use. Moreover, increasing resistance to both these agents has been reported. Therefore, empiric regimens suggested for nosocomial infections, also in countries like Italy, in which the incidence of CRKP is not negligible, are mostly ineffective against CRKP.

The paucity of therapeutic options emphasizes the need for new treatments. The combinations of avibactam, a new $\beta$-lactamase-inhibitor, with $\beta$-lactams or monobactams such as ceftazidime, ceftaroline or aztreonam, could represent new alternatives to carbapenems. Other drugs in clinical development are plazomicin (a novel aminoglycoside) and eravacycline (a new tetracycline), active against most KPC-producing Enterobacteriaceae..$^{40,41}$

Whenever available in microbiological laboratories, the new rapid molecular assays already licensed for commercial use, are of great utility to ensure an earlier effective treatment of MDR infections, and in particular of CRKP infections, with specific agents. The matrix-assisted laser desorption/ionization time of flight mass spectrometry is a fast and cost-effective test in which the mass-charge ratios of molecular fragments obtained by ionization and disintegration of a target molecule are compared with those of known microorganisms. Although the test does not provide data on antimicrobial susceptibility, the time to effective therapy is reduced from 89.7 to $32 \mathrm{~h}(\mathrm{P}=0.001){ }^{42,43}$ Multiplex polymerase chain reaction (PCR) and nucleic acid extraction and PCR amplification techniques, allow the detection of multiple organisms and resistance markers within two hours from blood cultures positivity. ${ }^{44,45}$ These new microbiological technologies allowing the rapid identification of resistant strains and the early beginning of specific effective therapy, can really modify the outcome of severe infections caused by MDR strains as CRKP.

\section{Key message}

Antibiotic regimens active against Gram-negative bacilli: i) ESBL-: $3^{\text {rd }}$ generation cephalosporins, monobactam, piperacillin-tazobactam; ii) ESBL+: (mild or moderate, or urinary tract infections): piperacillintazobactam, carbapenems; iii) ESBL+: (severe infections); carbapenems; iv) CRKP: colistin, carbapenems, tigecyclin, aminoglycosides (in combination therapy). 


\section{Outcomes of carbapenem-resistant Klebsiella pneumoniae sepsis}

CRKP infections are associated with a mortality of $40-50 \%{ }^{46,47}$ Most deaths occur within two weeks from the infection onset. ${ }^{48}$ In cancer patients, the reported 30 -day mortality is higher $(57.8 \%$ for all infections, $72.7 \%$ for bacteremic infections). ${ }^{49}$

In 2012 Lee and Burgess reviewed the outcome of 105 patients with infection by KPC-producing organisms reported in 54 published articles. The overall rate of treatment failure was $36 \%$; pulmonary infections and bloodstream infections were associated with the highest rate of treatment failure ( $47 \%$ and $39 \%$ respectively). ${ }^{50}$

The poor prognosis associated with MDR infections probably is not related to increased virulence of the pathogens. Viale et al. recognized several risk factors predictive of a poor outcome: patient-related factors (age, comorbidities, APACHE score), infection-related factors (bloodstream or pulmonary source of infection, severe sepsis or septic shock, colistin-resistant strain) and treatment-related factors (not effective empiric treatment, monotherapy). ${ }^{40}$ The lack of initially appropriate antibiotic therapy seems to be the major determinant of outcome in severe infections. In a recent series of 1076 patients with severe sepsis or septic shock due to Gram-negative pathogens, 5.9\% of the isolates were MDR; overall in-hospital mortality was $29.2 \%$. The rate of MDR infections was $10 \%$ in the non-survivor group and $4 \%$ in the survivor group ( $\mathrm{P}=0.001)$; the rate of inappropriate empiric treatment was $43.4 \%$ and $14.6 \%(\mathrm{P}=0.001)$ respectively. The inappropriate initial empiric therapy was an independent predictor of hospital mortality (adjusted OR 3.87) and MDR resulted strongly associated with inappropriate initial treatment (adjusted OR 13.05). ${ }^{11}$ Indeed, the empiric antibiotic regimens usually selected for early treatment of nosocomial infections are not effective against CRKP.

The therapeutic options for CRKP are very limited and include colistin, tigecycline and aminoglycosides; meropenem is useful for in vitro resistant strains with MIC values $\leq 8$. The results obtained with these agents in monotherapy and combination therapy were reviewed by Lee and Burgess ${ }^{50}$ and are reported in Table 3 .

In the therapy of CRKP infections, colistin is the most commonly used agent and is considered as the last resort. However, the effectiveness of colistin is not well established and colistin monotherapy had high rate of therapeutic failure (73\%). Moreover, colistinresistance, although infrequently, has been reported and could be the last step towards pan-drug resistance (resistance to all available antibiotics) of K. pneumoniae. ${ }^{5}$ Monotherapy with carbapenems was also associated with frequent failures $(60 \%)$.

Combination therapy is more effective than monotherapy, with rates of therapeutic failures of $29 \%$ with colistin-including combination regimens and $26 \%$ with carbapenem-including combination regimens. ${ }^{50}$ Combination regimens including meropenem at high dosage (4-6 g die) seem associated with better outcome. ${ }^{51,52}$ High doses of tigecycline (100 mg every $12 \mathrm{~h}$ ) have been reported to be superior to standard doses in combination regimens for pulmonary infections. ${ }^{53}$ However, emergence of isolates with reduced susceptibility to tigecycline has been reported. ${ }^{54}$ Nearly $40 \%$ of CRKP are resistant to colistin as well as to meropenem; for these strains combination regimens including gentamicin seem to be associated with better outcome. ${ }^{55}$ Combinations of colistin plus rifampin, meropenem plus ertapenem or meropenem plus ertapenem plus colistin have been also proposed for the treatment of infections caused by these extremely drug resistant strains. ${ }^{56,57}$

\section{Key messages}

- Mortality: 40-50\%

- Risk factors for poor outcome: i) patient-related (age, comorbidities, APACHE score); ii) infection-related (blood or pulmonary source, severity, colistin-resistance); iii) treatment-related (monotherapy, deleted effective treatment).

Table 3. Treatment failure: monotherapy versus combination therapy.

\begin{tabular}{|c|c|c|c|c|}
\hline \multicolumn{2}{|c|}{ Treatment failure } & \multirow{2}{*}{$\begin{array}{c}\text { Monotherapy (\%) } \\
14 / 56(25)\end{array}$} & \multirow{2}{*}{$\begin{array}{c}\text { Combination therapy }(\%) \\
0.01\end{array}$} & \multirow[t]{2}{*}{$\mathbf{P}$} \\
\hline Overall & $24 / 49(49)$ & & & \\
\hline \multirow[t]{3}{*}{ Source: } & - blood & $12 / 24(50)$ & $9 / 32(28)$ & 0.09 \\
\hline & - pulmonary & $10 / 15(67)$ & $5 / 17(29)$ & 0.03 \\
\hline & - urine & $1 / 8(13)$ & $0 / 3(0)$ & 0.4 \\
\hline Colistin & 8/11 (73) & $10 / 34(29)$ & 0.02 & \\
\hline \multicolumn{2}{|c|}{ Carbapenem } & $12 / 20(60)$ & $5 / 19(26)$ & 0.03 \\
\hline \multicolumn{2}{|c|}{ Tigecycline } & 2/7 (29) & $7 / 19(37)$ & 0.4 \\
\hline \multicolumn{2}{|c|}{ Aminoglycoside } & $0 / 6(0)$ & 4/24 (17) & 0.6 \\
\hline
\end{tabular}

Data from Lee and Burgess, $2012 .{ }^{50}$ 


\section{Conclusions}

One third of HAIs are caused by Gram-negative bacteria and $K$. pneumoniae represents the species more involved in severe infections, such as pneumonia, bloodstream infections and ICU infections. In Italy, the incidence of resistant strains among nosocomial isolates of $K$. pneumoniae is very high: almost two thirds of isolates are resistant to third generation cephalosporins and half of the isolates are MDR. The major contribute to selection of resistant strains is the antibiotic pressure due to use of these agents. On the other hand, the main positive prognostic factor for severe infections is the initially appropriate antibiotic treatment, started empirically, immediately after microbiological cultures have been performed. In the era of ESBL-producing Enterobacteriaceae, the antibiotic regimens suggested for severe HAIs are necessarily based on carbapenems and the only possibility to contain the exposure to these agents is the early adoption of the de-escalation therapy, switching to other classes of antibiotics on the basis of the microbiological results documenting the absence of resistant pathogens.

In this context, carbapenem-resistant Gram negative are spreading in our hospitals. In particular, more than one third of $K$. pneumoniae isolates are actually carbapenem-resistant.

To reduce the trend of further increase in resistance, antimicrobial stewardship programs are essential to promote carbapenem-sparing treatments. The selection of empiric regimens should be based on the evaluation of risk factors of each patients and on the severity of infection. For patients admitted to the hospital with apparently community-acquired infection, the evaluation of risk factors for MDR infections (previous hospitalizations, residence in nursing-home, recent antibiotic treatments) is mandatory. A decisional approach based on calculation of risk-scores has been validated for health-care associated pneumonia, but is still under evaluation for other HCAIs. Different empiric regimens are required for patient without risk factors for resistant strains (community acquired infections), patients with risk factors for MDR and mild to moderate infections or urinary tract infections (carbapenem-sparing regimens, with preference for ureidopenicillin plus $\beta$-lactamase inhibitor) and patients with severe infections or risk factors for CRKP infections (carbapenem-based regimens).

Although patients at high risk for CRKP can be identified on the basis of specific risk factors (Charlson score $\geq 3$, two or more hospitalizations in the previous year, treatment with carbapenems or fluoroquinolones in the previous month, neutropenia, central venous catheter, enteric colonization by CRKP, previous $C$. difficile infections), initial empiric treatment effective against CRKP is not suggested. The nephrotoxicity of colistin, the lack of anti-Pseudomonas activity of tigecycline and the risk of selection of further resistances, make these agents not suitable for empiric usage. New promising antibiotics, actually under clinical investigations, could modify the current approach. In the meantime, the new rapid molecular assays in addition to traditional microbiological tests should be particularly useful to treat earlier these patients.

The only agents active against CRKP are colistin, tigecycline and aminoglycosides; meropenem can be used for resistant strains with MIC values $\leq 8$, accounting for about $30 \%$ of CRKP. The combination of these agents resulted superior to monotherapy and should be preferred.

\section{References}

1. Peleg AY, Hooper DC. Hospital-acquired infections due to Gram-negative bacteria. N Engl J Med 2010;362:1804-13.

2. Gaynes R, Edwards JR. Overview of nosocomial infections caused by Gram negative bacilli. Clin Infect Dis 2005;41:848-54.

3. European Center for Disease Prevention and Control (ECDC). Surveillance report - Annual epidemiological report 2014 - Antimicrobial resistance and healthcare-associated infections. Stockholm: ECDC; 2015. Available from: http://ecdc.europa.eu/en/publications/publications/ antimicrobial-resistance-annual-epidemiologicalreport.pdf

4. Pitout JD, Laupland KB. Extended-spectrum beta-lactamase-producing Enterobacteriaceae: an emerging public-health concern. Lancet Infect Dis 2008;8:159-66.

5. European Center for Disease Prevention and Control (ECDC). Antimicrobial resistance surveillance in Europe 2014. Annual Report of the European Antimicrobial Resistance Surveillance Network (EARS-Net). Stockholm: ECDC; 2015. Available from: http://ecdc. europa.eu/en/publications/publications/antimicrobial-resistance-europe-2014.pdf

6. Kollef MH, Sherman G, Ward S, Fraser VJ. Inadequate antimicrobial treatment of infections: a risk factor for hospital mortality among critically ill patients. Chest 1999; 115:462-74.

7. Peraita G, Lamelo M, Alvarez-Garcia P, et al. Impact of empirical treatment in extended-spectrum beta-lactamase-producing Escherichia coli and Klebsiella spp. bacteremia. A multicentric cohort study. BMC Infect Dis 2012;12:245.

8. Hidron AI, Edwards JR, Patel J, et al. NHSN annual update: antimicrobial-resistant pathogen associated with healthcare associated infections: annual summary of data reported to the National Healthcare Safety Network at the Centers for Disease Control and Prevention, 2006-2007. Infect Control Hosp Epidemiol 2008;29:996-1011.

9. Yigit H, Queenan AM, Anderson GJ, et al. Novel carbapenem-hydrolyzing beta-lactamase, KPC-1, from a carbapenem-resistant strain of Klebsiella pneumoniae. Antimicrob Ag Chemother 2001;45:1151-61.

10. Bradford PA, Bratu S, Urban C, et al. Emergence of car- 
bapenem-resistant Klebsiella species possessing the class A carbapenem-hydrolyzing KPC-2 and inhibitorresistant TEM-30 beta-lactamases in New York City. Clin Infect Dis 2004;39:55-60.

11. Zilberberger MD, Shorr AF, Micek ST, et al. Multi-drug resistance, inappropriate initial antibiotic therapy and mortality in Gram-negative severe sepsis and septic shock: a retrospective cohort study. Critical Care 2014;18: 596-608.

12. Boontham P, Soontomrak R. Intra-abdominal infections: prevalence and risk factors of ESBL infections. J Med Assoc Thai 2015;98:1097-103.

13. McKay R, Bamford C. Community versus healthcare-acquired bloodstream infections at Groote Schuur Hospital, Cape Town, South Africa. S Afr Med J, 2015; 105:363-9.

14. Center for Disease Control and Prevention. Vital signs: carbapenem-resistant Enterobacteriaceae. MMWR Morb Mort Wkly Rep 2013;62:165-70.

15. Kollef MH, Shorr A, Tabak YP et al. Epidemiology and outcomes of health-care-associated pneumonia: results from a large US database of culture-positive pneumonia. Chest 2005;128:3854-62.

16. American Thoracic Society; Infectious Diseases Society of America. Guidelines for the management of adults with hospital-acquired, ventilator-associated and healthcare-associated pneumonia. Am J Respir Crit Care Med 2005;171:388-416.

17. Venditti M, Falcone M, Corrao S, et al. Outcomes of patients hospitalized with community-acquired, health care-associated and hospital-acquired pneumonia. Ann Intern Med 2008;150:19-26.

18. Shorr AF, Zilberberger MD, Micek ST, et al. Prediction of infections due to antibiotic-resistant bacteria by select risk factors for healthcare-associated pneumonia. Arch Intern Med 2008;168:2205-10.

19. Nseir S, Grailles G, Soury-Lavergne A et al. Accuracy of American Thoracic Society/Infectious Disease Society of America criteria in predicting infection or colonization with multidrug-resistant bacteria at intensive-care unit admission. Clin Microbiol Infect 2010; 16:902-8.

20. Shindo Y, Ito R, Kobayashi D, et al. Risk factors for drug-resistant pathogens in community-acquired and healthcare-associated pneumonia. Am J Respir Crit Care Med, 2013;188:985-95.

21. Maruyama T, Fujisawa T, Okuno M, et al. A new strategy for healthcare-associated pneumonia: a 2-year prospective multicenter cohort study using risk factors for multidrug-resistant pathogens to select initial empiric therapy. Clin Infect Dis 2013;57:1373-83.

22. Falcone M, Russo A, Giannella M, et al. Individualizing risk of multidrug-resistant pathogens in communityonset pneumonia. PLoS One 2015;10:1371.

23. Pop-Vicas A, Mitchell SI, Kandel R, et al. Multidrugresistant Gram-negative bacteria in a long-term care facility: prevalence and risk factors. J Am Geriatr Soc 2008;56:1276-80.

24. Kothe H, Bauer T, Marre R, et al. Outcome of community-acquired pneumonia: influence of age, residence status and antimicrobial treatment. Eur Respir J 2008; 32:139-4.

25. Costelloe C, Metcalfe C, Lovering A, et al. Effect of antibiotic prescribing in primary care on antimicrobial re- sistance in individual patients: systematic review and meta-analysis. BMJ 2010;340:c2096.

26. Pop-Vicas A, Strom J, Stanley K, et al. Multidrug-resistant Gram-negative bacteria among patients who require chronic hemodialysis. Clin J Am Soc Nephrol 2008;3: 752-8.

27. Akova M, Daikos GL, Tzouvelekis L, Carmeli Y. Interventional strategies and current clinical experience with carbapenemase-producing Gram-negative bacteria. Clin Microbiol Infect 2012;18:439-48.

28. Tumbarello M, Trecarichi EM, Tumietto F et al. Predictive model for identification of hospitalized patients harboring KPC-producing Klebsiella pneumoniae. Antimicrob Agents Chemother 2014; 58:3514-20.

29. Ling ML, Tee YM, Tan SG. Risk factors for acquisition of carbapenem resistant Enterobacteriaceae in an acute tertiary care hospital in Singapore. Antimicrob Resist Infect Control 2015;4:26-32.

30. Papadimitriou-Olivgeris M, Marangos M, Fligou F, et al. Risk factors for KPC-producing Klebsiella pneumoniae enteric colonization upon ICU admission. J Antimicrob Chemother, 2012; 67:2976-81

31. Papadimitriou-Olivgeris M, Marangos M, Fligou F et al. KPC-producing Klebsiella pneumoniae enteric colonization acquired during intensive care unit stay: the significance of risk factors for its development and its impact on mortality. Diagn Microbiol Infect Dis 2013;77:169-73.

32. Feldman N, Adler A, Molshatzki N, et al. Gastrointestinal colonization by KPC-producing Klebsiella pneumoniae following hospital discharge: duration of carriage and risk factors for persistent carriage. Clin Microbiol Infect 2013;19:E190-96.

33. Wiener-Well Y, Rudensky B, Yinnon AM et al. Carriage rate of carbapenem-resistant Klebsiella pneumonia in hospitalized patients during a national outbreak. J Hosp Infect 2010;74:344-9.

34. Borer A, Saidel-Odes L, Eskira S, et al. Risk factors for developing clinical infection with carbapenem-resistant Klebsiella pneumoniae in hospital patients initially only colonized with carbapenem-resistant Klebsiella pneumoniae. Am J Infect Control 2012;40:421-5.

35. Prasad N, Labaze G, Kopacz J, et al. Asymptomatic rectal colonization with carbapenem-resistant Enterobacteriaceae and Clostridium difficile among residents of a long-term carefacility in New York City. Am J Infect Control 2016 [In press].

36. Lubbert C, Faucheux S, Becker-Rux D, et al. Rapid emergence of secondary resistance to gentamicin and colistin following selective digestive decontamination in patients with KPC-2-producing Klebsiella pneumoniae: a single-centre experience. Int J Antimicrob Agent 2013;42:565-70.

37. Saidel-Odes L, Polachek H, Peled N, et al. A randomized, double-blind, placebo-controlled trial of selective digestive decontamination using oral gentamicin and oral polimyxin E for eradication of carbapenem-resistant Klebsiella pneumoniae carriage. Infect Control Hosp Epidemiol 2012;33:14-9.

38. Oren I, Sprecher H, Finkelstein R, et al. Enterobacteriaceae gastrointestinal colonization with nonadsorbable oral antibiotic treatment: a prospective controlled trial. Am J Infect Control 2013;41:1167-72. 
39. Felton TW, Hope WW, Lomaestro BM, et al. Population pharmacokinetics of extended-infusion piperacillintazobactam in hospitalized patients with nosocomial infections. Antimicrob Agents Chemother 2012;56:4087-94.

40. Viale P, Giannella M, Bartoletti M, et al. Considerations about antimicrobial stewardship in setting with epidemic extended-spectrum beta-lactamase-producing or carbapenem-resistant Enterobacteriaceae. Infect Dis Ther 2015;4:S65-83.

41. Munoz-Price LS, Poirel L, Bonomo RA, et al. Clinical epidemiology of global expansion of Klebsiella pneumoniae carbapenemases. Lancet Infect Dis 2013;13:785-96.

42. Tan KE, Ellis BC, Lee R, et al. Prospective evaluation of a matrix-assisted laser desorption ionization-time of flight mass spectrometry system in a hospital clinical microbiology laboratory for identification of bacteria and yeasts: a bench-by-bench study for assessing the impact on time to identification and cost-effectiveness. J Clin Microbiol 2012;50:3301-8.

43. Clerc O, Prod'hom G, Vogne C, et al. Impact of matrixassisted laser desorption ionization time-of-flight mass spectrometry on the clinical management of patients with Gram negative bacteremia: a prospective observational study. Clin Infect Dis 2013;56:1101-7.

44. Mancini N, Infurnari I, Ghidoli N, et al. Potential impact of a microarray-based nucleic acid assay for rapid detection of Gram negative bacteria and resistance markers in positive blood cultures. J Clin Microbiol 2014;52:1242-5.

45. Bork JT, Leekha S, Heil EI, et al. Rapid testing using the Verigene Gram-negative blood culture nucleic acid test in combination with antimicrobial stewardship intervention against Gram-negative bacteremia. Antimicrob Agents Chemother 2015;59:1588-95.

46. Bratu S, Landman D, Haag R, et al. Rapid spread of carbapenem-resistant Klebsiella pneumoniae in New York City: a new threat to our antibiotic armamentarium. Arch Int Med 2005; 165:1430-5.

47. Viale P, Giannella M. Lewis R et al. Predictors of mortality in multidrug-resistant Klebsiella pneumoniae bloodstream infections. Expert rev Anti-infect Ther 2013;11: 1053-63.
48. Tumbarello M, Trecarichi EM, De Rosa FG, et al. Infections caused by KPC-producing Klebsiella pneumoniae: differences in therapy and mortality in a multicenter study. J Antimicrob Chemother 2015;70:2133-43.

49. Freire MP, Pierotti LC, Filho HH, et al. Infection with Klebsiella pneumoniae carbapenemase (KPC)-producing Klebsiella pneumoniae in cancer patients. Eur J Clin Microbiol Infect Dis 2015;34:277-86.

50. Lee CG, Burgess DS. Treatment of Klebsiella pneumoniae Carbapenemase (KPC) infections: a review of published case series and case reports. Ann Clin Microbiol Antimicrob 2012;11:32-38.

51. Tumbarello M, Viale P, Viscoli C. Predictors of mortality in bloodstream infections caused by Klebsiella pneumoniae carbapenemase-producing K. pneumoniae: importance of combination therapy. Clin Infect Dis 2012;55:943-50.

52. Daikos GI, Tsapusi S, Tzouvelekis LS, et al. Carbapenemase-producing Klebsiella pneumoniae bloodstream infections: lowering mortality by antibiotic combination schemes and the role of carbapenem. Antimicrob Agents Chemother 2014;58:2322-8.

53. De Pascale G, Montini L, Pennisi M, et al. High dose tigecycline in critically ill patients with severe infections due to multidrug resistant bacteria. Crit Care 2014;18:R90.

54. Van Duin D, Cober ED, Richter SS, et al. Tigecycline therapy for carbapenem-resistant Klebsiella pneumoniae (CRKP) bacteriuria leads to tigecycline resistance. Clin Microbiol Infect 2014;20:O1117-20.

55. Gonzalez-Padilla M, Torre-Cisneros J, Rivera Espinar F, et al. Gentamicin therapy for sepsis due to carbapenem-resistant and colistin-resistant Klebsiella pneumoniae. J Antimicrob Chemother 2015;70:905-13.

56. Tascini C, Tagliaferri E, Giani T, et al. Synergistic activity of colistin plus rifampin against colistin-resistant KPC-producing Klebsiella pneumoniae. Antimicrob Agents Chemother 2013;57:3990-3.

57. Bulik CC, Nicolau DP. Double-carbapenem therapy for carbapenemase-producing Klebsiella pneumoniae. Antimicrob Agents Chemother 2011;55:3002-4. 